\title{
Rowing and the Same-Sum Problem Have Their Moments
}

\author{
John D. Barrow \\ DAMTP, Centre for Mathematical Sciences, \\ Cambridge University, \\ Wilberforce Rd., Cambridge CB3 0WA, UK
}

August 17, 2010

\begin{abstract}
We consider the optimal positioning of an even number of identical crew members in a coxless racing boat in order to avoid the presence of a sideways wiggle as the boat is propelled forwards through the water. We show that the traditional (alternate port and starboard) rig of racing boats always possesses an oscillating non-zero transverse moment and associated wiggling motion. We show that the problem of finding the zero-moment rigs is related to a special case of the Subset Sum problem. We find the one (known) zero-moment rig for a racing Four and show there are four possible such rigs for a racing Eight, of which only two (the so called 'Italian' and 'German' rigs) appear to be already known. We also give the 29 zero-moment solutions for racing Twelves but refrain from explicitly listing the 263 Sixteens and 2724 Twenties which have zero transverse moments. We show that only balanced boats with crew numbers that are divisible by four can have the zero-moment property. We also discuss some aspects of unbalanced boats, in which the number of port and starboard oars are unequal.
\end{abstract}

\section{$1 \quad$ Messing About in Boats}

In this article we are going to consider some simple mathematical properties of (heavily idealized) coxless rowing crews. Specifically, we are going to investigate how the placement of the rowers (the 'rig') of the boat has some simple dynamical consequences for the motion of the boat. The traditional way of rigging a boat, places alternates rowers pulling oars on the two sides of the boat, as in Figure 1 for a Four. We will label this traditional rig configuration of oarsmen 


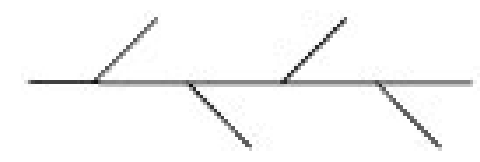

Figure 1: The traditional rig of a Four, which we label $u d u d$.

as $u d u d$ where $u$ and $d$ label the two sides of the boat (up and down as we look at the page but starboard and port when viewed from inside the boat).

If we look at the boat from the other side then it will have the mirror configuration $d u d u$. We regard these as identical and do not distinguish rigs under the relabelling transformation $(u, d) \longleftrightarrow(d, u)$.

For simplicity we are going to make some assumptions. All our rowers are assumed to be identical in strength and technique and to employ identical equipment with identical set up. They will each pull only a single oar on one side of the boat (no double scullers). We will not include a cox or any other influence (rowers of unequal strengths) that might counteract sideways forces. All of these simplifications can be dropped if necessary and adapted versions of our conclusions will hold. Finally, unless we say otherwise we will always we considering crews with the same number of oars pulling on each side of the boat. We will call such a boat a balanced boat.

The traditional rig appears symmetrical and simple in ways that might tempt you into thinking it is in every sense optimal. However, this is not the case, as we shall now see.

\section{The Moment Problem}

First, consider the set-up of a balanced Four. The standard rig for this boat has a simple alternation of rowers which we shall call the standard rig, shown in Figure 1. A time-varying force is exerted on the boat by the torque applied by each rower's oar in its rowlock. The time variation of this 'rowing force curve' is much studied by coaches seeking to balance crews, improve technique, and understand the strengths and weaknesses of crew members individually and collectively [1. We shall ignore all this detailed analysis together with details of blade shape, angle to the water, flexure and hydrodynamics [2, and note simply that the force can always be resolved into its two perpendicular components: one in the direction of the boat's forward motion and the other at right angles 


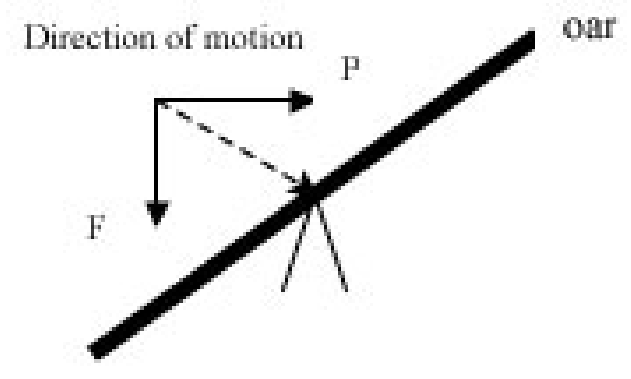

Figure 2: The orthogonal components of the force exerted on the boat at the oarlock by an oar. During the first phase of the stroke the component normal to the direction of motion, $F$, is directed towards the boat. The component in the direction of motion is $P$.

to it. We shall call the latter normal component to the line through the stern and the bow the transverse force.

During the first (catch and drive) part of the rower's stroke the transverse force at the oarlock is directed towards the boat (see Figure 2) but during the second (extraction and recovery) phase of the stroke it reverses and is directed away from the boat (see Figure 3) [3]. This alternation in the direction of the transverse component of the force exerted on the boat continues almost periodically as the rowers repeat their sequence of stroke patterns.

Let us now sum the moments of these transverse forces about any point on the centre line of the boat and we will pick that point to be the stern. Assume the distance from the stern to the first rower (the stroke) is $s$ and the spacing between all the other three rowers in the Four is $x$. During the first phase of their synchronised strokes assume that each rower exerts the same transverse force of magnitude $F>0$. The net sideways moment on the traditionally rigged $u d u d$ boat is equal to

$$
M(\text { phase } 1)=s F-(s+x) F+(s+2 x) F-(s+3 x) F=-2 F x<0
$$

where we have picked the 'up direction in our diagram for the + sign.

There are three simple points to notice from this result for the traditionally rigged crew of the Four. First, because our crews will always be balanced, with the same number of oars on each side, the dependence on the distance to the stroke's location, $s$, always cancels out and we can set it to any value we choose and henceforth we set $s=1$. Second, the value of $x$ can also be set equal to unity 


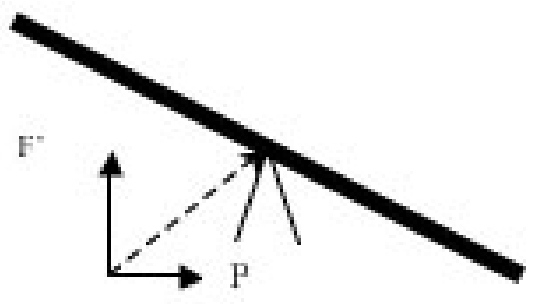

Direction of motion

oar

Figure 3: During the second (recovery) phase of the stroke the component of the force normal to the direction of motion, $F^{\prime}$, is reversed and points away from the boat. The component in the direction of motion, $P$, keeps the same sign.

by absorbing it into $F$ or $F^{\prime}$ by a redefinition. Third, and most important, the net moment on the boat during the first phase of the stroke with the traditional rig is non-zero. During the second recovery phase of the stroke the sign (and possibly the value) of the transverse force will change to $-F^{\prime}<0$ and the net moment on the boat will now be

$$
M(\text { phase } 2)=+2 F^{\prime} x>0
$$

As a result, the boat will wiggle steadily from side to side as it moves forward under the influence of this alternating transverse force. It can be countered by the rowers (or by a cox) sensing its existence and making small adjustments. But this takes extra energy from the rowers and slows the forward progress of the boat. Ideally, we might like to eliminate this transverse alternating torque on the boat. This wiggle will arise whenever there is an asymmetry in the force applied by a rower during the whole stroke cycle, even if there is no change of sign in the normal component. In fact, in our model, whenever $F \neq F^{\prime}$ there we will be a sideways drift of the boat in addition to a wiggle. For simplicity, we shall take $F=F^{\prime}$ in what follows. Although this is not entirely realistic, it does not affect our results because we are going to be interested in finding situations where the net moment on the boat vanishes. From now on we will also set $x \equiv s \equiv F \equiv F^{\prime} \equiv 1$.

Let's stay with the possible balanced rigs for the Four. There are only three distinct possibilities, shown in Figure 4. 
(a)

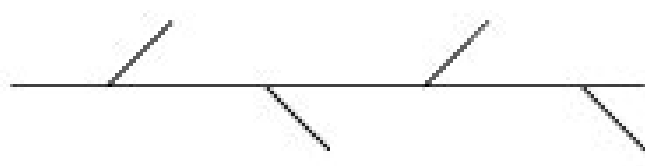

(b)

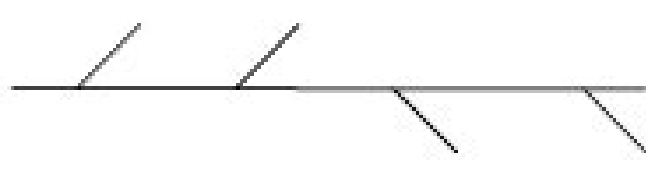

(c)

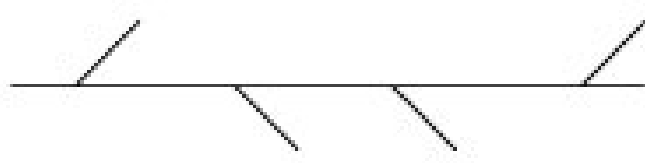

Figure 4: The three distinct ways to rig a Four: (a) The traditional rig, udud, with transverse moment \pm 2 ; (b) The worst rig, uudd, with transverse moment \pm 4 ; (c) The 'Italian' rig, uddu, with zero transverse moment.

We can swop the $u$ and $d$ positions but that is just a mirror of the original (eg $u d u d$ and $d u d u$ are both the standard rig) and we don't count.

The standard rig (a) has a non-zero moment and a wiggle with $M= \pm 2$. The rig (b) is the worst way to rig a Four, with a transverse moment that is twice that of the standard rig:

$$
\pm M=1+2-3-4=-4 .
$$

However, the last possibility, (c), has a zero moment ,

$$
\pm M=1-2-3+4=0
$$

and for this configuration there will be no wiggle in the boat's forward motion. This configuration is known as the 'Italian' rig because it was discovered by the Moto Guzzi Club team on Lake Como in 1956. The crew of the Club's Four was being watched by Giulio Cesare Carcano, one of the company's leading motorcycle engineers from Milan, who suggested that the failure of the boat to run straight might be alleviated by putting the middle two oarsmen both on the starboard side with the stroke and the bow still on the port side [4]. The result was rather successful and the Moto Guzzi crew went on to represent Italy and take the Gold Medal that year at the Melbourne Olympic Games. 


\section{A 'Momentous' General Formulation}

We can see from the simple example of the Four that the values the transverse moments during each of the two phases of the stroke are given by

$$
\begin{aligned}
& \text { (a) : udud : } 1-2+3-4=-2 \\
& \text { (b) : uudd : } 1+2-3-4=-4 \\
& \text { (c) : uddu : } 1-2-3+4=0
\end{aligned}
$$

The zero-moment rig is the one in which the sum of the first 4 integers can be combined with 2 plus signs and 2 minus signs, as required for balance, so that their sum is zero. The first entry, +1 , will always be taken positive without any loss of generality because the case with -1 will just produce the mirror rig.

This formulation allows us to look at the generalisations to balanced crews containing with zero transverse moment with any even number of rowers. If there are $2 N$ rowers, with $N=1,2,3, \ldots$ then we are looking for the set of solutions to the arithmetic problem

$$
1 \pm 2 \pm 3 \pm \ldots \ldots . \pm 2 N=0
$$

in which the left-hand side contains $N$ plus signs and $N$ minus signs.

We can immediately draw a simple conclusion by using this formulation:

\section{Balanced zero-moment rigs are only possible with $2 N$ rowers if $\mathrm{N}$ is even;}

that is, the number in the crew must be divisible by 4 . Thus, there cannot be a balanced Six with zero transverse moment. The proof is simple: we have to add or subtract $N$ even numbers and $N$ odd numbers to get zero. This is only possible if $N$ is even because only then will the combination of the $N$ odd numbers be even like the combination of the $N$ even numbers and only then can the sum of both be zero. The simplest case of all, that of a pair $(N=1)$ also always has a non-zero moment equal to $1-2=-1$, or $+1=-1+2$ for the mirror rig. Hence, we can now confine our attention to crews of 4,8 , 12, etc.

We can streamline the formulation in a further interesting fashion by noting that the sum requirement (2) for a zero moment is equivalent to the requirement that the sum of all the numbers from 1 to $2 N$ is equal to twice the sum of all those entering with + signs (or minus all those entering with minus signs):

$$
\sum_{r=1}^{2 N} r+2\left(\sum \text { negative entries }\right)=0=\sum_{r=1}^{2 N} r-2\left(\sum \text { positive entries }\right)
$$

where the second sum is just over the $N$ entries with negative (or positive) signs, respectively. This means, for a crew of $2 N$ rowers, 
$N(2 N+1)+2\left(\sum\right.$ negative entries $)=0=N(2 N+1)-2\left(\sum\right.$ positive entries $)$.

So we just need to find the cases where the sum of the $N$ negative (or positive) entries add up to

$$
\frac{N(2 N+1)}{2}
$$

For the case of the Four $(N=2)$ we need the sum of the $N=2$ negative entries to be equal to -5 , which was indeed the case for the zero-moment configuration (c) given above in (1).

This problem is now recognisable as a version of the Subset-Sum problem in which a subset, $S_{k}$ of a set positive integers, $S$, is selected by the requirement that the sum of the members of the subset be equal to some integer, $k$. The version of this algorithmic problem that we are faced with here is the Same Sum problem [5] in which we want to find all the subsets $S_{k}$ of $S$ which have the same sum, $k=N(2 N+1) / 2$. Jeffrey Shallit has also pointed out a connection to structure of the Thue-Morse sequence [6].

\section{Eights, and After}

As with the Four, the traditional rig for a rowing Eight has a significant nonzero moment $(M= \pm 4)$ and a counter-productive transverse wiggle for the cox to counter. This non-zero moment corresponds to a non-zero sum in which the sum of the negative entries is -20 rather than the value -18 required for a non-zero moment because:

$$
\text { udududud: } 1-2+3-4+5-6+7-8=-4
$$

For the case of a rowing Eight, where $N=4$, the Same Sum problem for a zero-moment rig is the case where the sum of the magnitudes of the 4 negative entries the alternating sum must equal -18 . There are four distinct solutions to this problem:

$$
\begin{aligned}
& \text { (a) }: 3+4+5+6=18 \text { ie } u \text { udddduu } \\
& \text { (b) }: 2+4+5+7=18 \text { ie } u d u d d u d u \\
& \text { (c) }: 2+3+6+7=18 \text { ie } u d d u u d d u \\
& \text { (d) }: 2+3+5+8=18 \text { ie } u d d u d u u d
\end{aligned}
$$

These (together with their four mirrors) give the four possible zero-moment rigs for an Eight shown in Figure 5.

Two of these are known. Rig (c) is the so called 'Italian' or 'triple tandem' rig, that was used by the Italian eights in the 1950s as an extension of Carcano's 
(a)

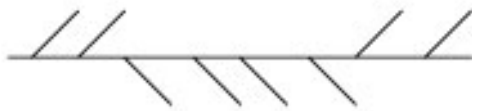

(b)

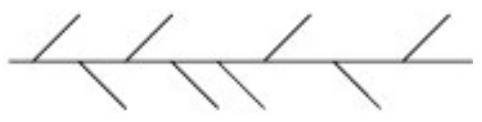

(c)

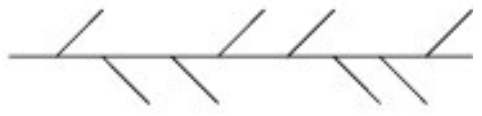

(d)

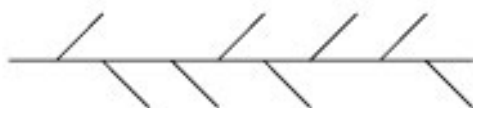

Figure 5: The four possible rigs for Eights which have zero transverse moment. (a) The New rig 1: uudddduu; (b) The 'German' rig: ududdudu; (c) The 'Italian' rig: udduuddu; and (d) The New rig 2: udduduud.

insight about the Four by adding two zero-moment (uddu) Fours together in series. Rig (b) is the so called 'German', 'bucket', or 'Ratzeburg rig', first used by crews training at that famous German rowing club in the late 1950s under Karl Adam, who was motivated by Carcano's configuration. Both the German and Italian crews were successful with these two zero-moment Eights and the zero-moment Four at the 1958 European Championships and the German crews coached by Adam went on to be supremely successful over the next ten years.

The other two zero-moment rigs found here, (a) and (d), appear to be new and have not previously been discussed. Note that $(\mathrm{d})$ is a combination of a zero-moment Italian Four with a mirrored zero-moment Italian Four. The other new rig, (a) is special because it manages to have a quadruple tandem configuration with a same-side Four positioned inside two same-side pairs.

The zero-moment eights have simple construction from the three possible Fours. All zero-moment Eights can be made by adding Fours with moments $-4+4,-2+2,0+0$, and $0-0$, where the minus sign indicates the switch $u \leftrightarrow d$. These four combinations correspond to the solutions (a), (b), (c) and (d), above respectively.

If we increase the number of rowers the number of permutations grows quickly. There are 29 distinct zero-moment rigs for 12-person crews, 263 zeromoment 16-person crews and 2724 zero-moment 20-person crews. For example, all the 12-rower crews correspond to all the solutions of the Same Sum problem, eq. (3), for which six of the numbers in $\{1,2,3 \ldots, 12\}$ sum to 39 . Here are all of these 29 solutions for the 12 seater crews [7]. The numbers appearing in each sum give the positions of the $d$ rowers : 


$$
\begin{aligned}
39 & =4+5+6+7+8+9=3+5+6+7+8+10=3+4+6+7+9+10 \\
& =2+5+6+7+9+10=3+4+5+8+9+10 \\
39 & =2+4+6+8+9+10=1+5+6+8+9+10=2+3+7+8+9+10 \\
& =1+4+7+8+9+10=3+4+6+7+8+11 \\
39 & =2+5+6+7+8+11=3+4+5+7+9+11=2+4+6+7+9+11 \\
& =1+5+6+7+9+11=2+4+5+8+9+11 \\
39 & =2+3+6+8+9+11=1+4+6+8+9+11=1+3+7+8+9+11 \\
& =3+4+5+6+10+11=2+4+5+7+10+11 \\
39 & =2+3+6+7+10+11=1+4+6+7+10+11=2+3+5+8+10+11 \\
& =1+4+5+8+10+11=1+3+6+8+10+11 \\
39 & =1+2+7+8+10+11=2+3+4+9+10+11=1+3+5+9+10+11 \\
& =1+2+6+9+10+11 .
\end{aligned}
$$

Each, as always, has a mirror solution in which these numbers give the positions of the $u$ crew members. For example, the mirror of the first solution given in the list corresponds to $1+2+3+10+11+12=0$. Again, the form of these zero-moment solutions can be analysed as composites of Eights and Fours with moments that combine so as to sum to zero in all possible ways.

\section{Unbalanced boats}

We have ignored the situation of unbalanced boats so far. They have unequal numbers of oars in the water on the two sides of the boat and so there will always be an asymmetry in the total forward force on the boat on each side and it will veer away from its straight-line forward course unless corrective action is taken. However, it is possible to have a zero transverse moment in these cases under particular conditions. Because the boat is no longer balanced, the size of $s$, the distance from the bow to the first rower will matter. Consider a Three with the rower's stations at distances $s, s+x, s+2 x$ from the stern. If $s=x$, there is a rig (uud) with zero transverse moment (shown in Figure 6) since the total moment is now

$$
M=s F+(s+x) F-(s+2 x) F=(s-x) F
$$

Similarly, as shown in Figure 7, we can make an unbalanced Six with a uuudud configuration that has moment

$M=s F+(s+x) F+(s+2 x) F-(s+3 x) F+(s+4 x) F-(s+5 x) F=(2 s-x) F$

which will be zero is we pick $x=2 s$. This is a general feature of unbalanced zero-transverse moment boats. The spacing of the stroke and bow position from the ends of the boat is constrained by the separation of the other rowers. 


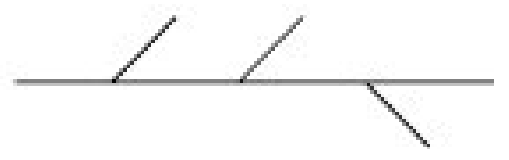

Figure 6: An unbalanced Three with $x=s$.

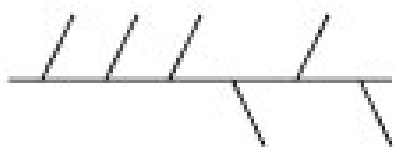

Figure 7: An unbalanced Six uuudud where $x=2 s$.

\section{Conclusions (and Apologies)}

Our idealized discussion of the transverse moments exerted by the crews of racing shells is not really intended to revolutionise the tactics adopted by worldclass racing crews (although we would be happy for that to be the case none the less). If it provides an instructive example of some simple mechanical principles in conjunction with an illustration of how a combinatorial problem arises in a familiar sporting context then it will have been sufficiently 'momentous'.

Acknowledgements I would like to thank Bill Atkinson, Baojiu Li, Sean Lip and Owen Smith for their assistance, and James Cranch and Jeffrey Shallit for helpful communications and references.

\section{References}

[1] For a summary by W. Atkinson see www.atkinssopht.com/row/forcpeak.htm. The most detailed study of rowing mechanics is quite old, by A. Dal Monte and A. Komar, Rowing and Sculling Mechanics in chapter 3 of C.L. Vaughan, ed. Biomechanics of Sport, CRC Press, Florida (1989), J.G.P. Williams and A.C. Scott, eds., Rowing - A Scientific Approach, A.S. Burns, NY, (1967) and D.L. Pope, in Mechanics and Sport, AMD-vol. 4, ed. J.L. Bleustein, Amer. Sci. Mech. Engineers, NY, (1973), pp. 267-275; M.N. Brearley, N.D. de Mestre and D.R. Watson, Math, Gazette 82, 389 (1998). 
[2] D. Cabrera, A. Ruina and V. Kleshnev, Human Movement Science 25, 192 (2006). M.N. Macrossan, The direction of the water force on a rowing blade, http://espace.library.uq.edu.au/eserv/UQ:134269/MER2008_03.pdf (2008)

[3] M. S. Townend, Teaching Mathematics 1, 18 (1982); A. Millward, J. Sports Sci. 5, 93 (1987); A.M. Wing and C. Woodburn, J. Sports Sci. 13, 187 (1995).

[4] A. Anderson, http://www.rowhistory.net/topic.asp?TOPIC_ID=116 quotation from a Rowing News article by A. Anderson. Other stories exist about the origin of the Moto Guzzi rig. One claims that the crew rigged the boat wrongly (probably deliberately) and their coach, suspecting that they were trying to avoid rowing a planned time trial told them they would have to do it in the mis-rigged boat and then do it again if they failed to make the target time. To everyone's surprise they surpassed both the target time and their own best performance over the course using their new rig.

[5] R.P. Stanley, SIAM J. Alg. Disc. Math. 1, 168 (1980) gives the first solution of such problems.

[6] J. Shallit, private communication.

[7] I am indebted to Sean Lip, for generating this list as well as its counterpart for larger boat crews which we do not include here because of limitations of space. 